\title{
Clinical undernutrition states and their influence on taste
}

\author{
H. I. M. Davidson*, R. M. Pattison and R. A. Richardson \\ Department of Dietetics and Nutrition, Queen Margaret College, Clerwood Terrace, Edinburgh EH12 8TS, UK
}

There are many sensory influences which originate in the gut and directly or indirectly influence nutritional status. However, those of which we are most consciously aware, such as taste and smell, are in most disease states not the first concern of most health-care professionals. This is because aberrations in these senses are not life-threatening, and the contribution they make to nutrient intake is considered to be relatively small in relation to the pathophysiological process alone. However, in conditions such as long-term chronic illness or neoplastic disease this may not be the case, as ingestive behaviour patterns may contribute to long-term energy deficit by dictating poor quality and quantity of nutrient intake. In addition, in conditions such as unresectable cancer where intervention is palliative only, the contribution that is made by somatic gustatory and olfactory aberrations may have increasing significance in terms of general quality of life, and have a significant detrimental effect not only on the patient but also on the carer. Elucidation of physiological or pathophysiological mechanisms which produce aberrant effects in these chemosensory pathways may provide the basis for more complete nutritional or palliative management, and reduce the negative impact that their dysfunction may have on nutritional status.

\section{Nutrient deprivation and taste acuity}

A number of studies have implicated undernutrition per se as a causative factor in producing alterations in taste. With the relatively high turnover rate of taste receptor cells and olfactory receptor cells these are likely to be affected more readily than others, with slow turnover rates in conditions where nutrient supplies for repair and replacement are scarce (Schiffman, 1994). A full review of the literature relating to the effects of undernutrition on taste acuity is outside the scope of the present paper. However, the contribution of selected nutritional deficiencies which may be most apparent in chronic disease states where undernutrition is a feature are discussed. Many of these studies are performed in experimental animals, and must be taken in context with the various degrees and types of gustatory and olfactory disturbances reported in human subjects (see Table 1).

The role of protein deficiency in taste sensitivity is well documented, particularly in animals which have not reached maturity (Vazquez et al. 1982; Ohara et al. 1994). This finding is not remarkable considering the increased energy demands for growth in young populations as a whole, and the fact that with low protein intakes protein turnover declines and therefore the likelihood of taste receptor cell dysfunction will increase. More recently Ohara et al. (1995) assessed the effect of not only different levels of dietary protein intakes, but also blood variables such as $\mathrm{Zn}$ considered to be involved in normal taste physiology in adult animals. Interestingly, in contrast to their earlier study (Ohara et al. 1994) the adult rats studied appeared fairly resistant to protein restriction in relation to taste sensitivity. Animals with no protein in their diet did have lower thresholds for salt however, but there was no complete blunting of taste preference. In addition, no differences were recorded in serum $\mathrm{Zn}$ concentrations between any of the protein-deficient groups.

There are a number of clinical conditions where low plasma $\mathrm{Zn}$ concentrations have been reported, including dysgeusia, hypogeusia and anorexia (Hambidge et al. 1972; Cohen et al. 1973). Indeed $\mathrm{Zn}$ supplementation was advocated, irrespective of the primary disorder, by some clinicians in patients with chemosensory abnormality. It appears that the effectiveness of such intervention relies on the cause of the aberration being entirely due to low concentrations of $\mathrm{Zn}$. The scarcity of results showing plasma $\mathrm{Zn}$ concentrations relating to degree of taste aberration is likely, in part, to be due to the fact that these measurements do not reflect the true amount of $\mathrm{Zn}$ available. Hair or nail sampling may provide a more reliable measurement of body stores, but even measurements taken from these samples fail to reveal any correlation with degree of taste acuity (Hambidge et al. 1972). In addition, the plasma concentrations of $\mathrm{Zn}$ are profoundly influenced by the presence of a metabolic response (Giacosa et al. 1996) such as that which occurs in infection or injury. This may also have a major role in complicating any interpretation of concentrations of $\mathrm{Zn}$ in plasma taste acuity.

Table 1. Nomenclature associated with chemosensory aberrations

\begin{tabular}{ll}
\hline Name & \multicolumn{1}{c}{ Meaning } \\
\hline $\begin{array}{l}\text { Normosmia } \\
\text { Normogeusia } \\
\text { Dysosmia }\end{array}$ & $\begin{array}{l}\text { Normal smell function } \\
\text { Normal taste function } \\
\text { Aberrant perception of smell either with or without } \\
\text { a stimulus } \\
\text { Aberrant sweet, sour, bitter or salt taste, with or } \\
\text { without the presence of metallic taste } \\
\text { Absence of smell } \\
\text { Anosmia }\end{array}$ \\
$\begin{array}{l}\text { Ageusia } \\
\text { Hypogeusia }\end{array}$ & $\begin{array}{l}\text { Reduced taste function, may be selective to one } \\
\text { taste modality }\end{array}$ \\
\hline
\end{tabular}


Thirty years ago, Bernard \& Halpern (1968) identified a role for vitamin A in taste transduction mechanisms. They showed that in comparison with normally-fed animals, those ingesting vitamin A-deficient diets had reduced baseline activity in the chorda tympani. In addition, activity evoked in the nerve after tastants were applied to the tongue was reduced by $50 \%$ in deficient animals. Considering the histological changes which occurred in this study, the conclusions of this group were that differences reported in tastant discrimination could be put down to the involvement of vitamin $\mathrm{A}$ in the mucopolysaccharide synthesis at the taste receptor level. However, the levels of deprivation may not represent any practical physiological relevance with regard to the degree of undernutrition necessitating such low intakes of vitamin A.

The lack of any single nutrient consistently providing a correlation with taste acuity probably indicates that the development of taste aberrations in undernutrition per se is multifactorial. In addition to which, a single measurement of a specific blood variable does not yield information on any physiologically relevant changes in these variables which may have occurred in the preceding period but have not yet reached a level defined as deficiency.

Without exception, in the studies reviewed previously in which macronutrient and micronutrient deficiencies exist, the associated taste change has resulted in a lack of discrimination between tastants or the requirement for an increase in concentration of tastant for apparently normal taste perception to occur. In disease states associated with undernutrition (where anorexia and/or cachexia may exist), dysgeusia and dysosmia may be common features of the disease or treatment modalities. Here the abberation is not always apparent as a loss of discrimination or reduction in tastant threshold. Patients will report simply that 'food does not taste the way it used to', or report the development of a disliking to tea or coffee. This may not exclusively reflect a reduction in chemosensory ability.

\section{Chemosensory disorders in disease}

The presence of chemosensory disturbances in disease states is a frequent phenomenon, but for the most part with unknown or no obvious aetiology. This may be compounded by the fact that these disorders are difficult to characterize and also because the patient may not recognize the sensory defect. Apparently simple descriptions of loss of taste and/or change in taste need to be substantiated initially, and in addition the associated loss of olfactory disturbances should be assessed, since subjective reporting of loss of taste usually turns out to be loss of olfaction (whether this be transient or permanent). Despite these recommendations, almost onequarter of certain chemosensory disturbances cannot be classified (Mott \& Leopold, 1991).

Although many chemosensory disorders can be directly attributed to diseases of the nose, sinuses, upper respiratory tract or the presence of head trauma, others have been reported to be secondary to the presence of undernutrition or the disease process per se. In addition, taste abnormalities can be induced by systemic and cognitive influences. Osaki et al. (1996) investigated patients presenting a variety of primary diagnoses (including xerostomia, anaemia and psychiatric distress) in an attempt to elucidate any common physiological variables which may be associated with the dysfunction. No biochemical variables were consistently shown to be abnormal in those with dysgeusia, but in those with hypogeusia $\mathrm{Fe}$ deficiency (but not $\mathrm{Zn}$ or $\mathrm{Cu}$ ) was accompanied by elevation of taste detection thresholds (TDT).

Disturbances of gustation and olfaction are common associated symptoms in clinical disease states such as neoplastic disease and hepatobiliary disorders. In the case of liver disease and primary tumours the pathophysiological processes causing the abberation are distant to the chemosensory transduction pathways. Possible mechanisms as to why they are still able to effect changes in taste and smell are discussed later (p. 636).

\section{Taste changes in cancer}

The evidence suggesting that taste changes and associated alterations in food preferences occur with the development of neoplastic disease is overwhelming (Vickers et al. 1981). This can occur irrespective of the location of the primary tumour or the concurrent (or previous) management with chemotherapy or radiotherapy regimens.

It is over 20 years since DeWys \& Walters (1975) noted alterations in TDT in cancer patients with a variety of tumour types. In particular, they noted elevated thresholds for sucrose and reduced thresholds for bitter; i.e. patients could taste bitter at lower concentrations than their non-cancer counterparts, but could only taste sucrose (sweet) at higher concentrations. This indicates that the taste abnormality found in cancer may not be the result of a simple nutrient deficiency-induced hypogeusia but that dysgeusia may occur simultaneously. In addition, this study was the first to highlight the association of aberration in taste occurring more readily in patients with significant weight loss.

In a similar study performed in a homogenous group of patients with regard to the primary tumour type, Williams \& Cohen (1978) measured taste thresholds in lung-cancer patients. Although the results were not as clear cut as those of DeWys \& Walters (1975), they also noted hypersensitivity to bitter in $10 \%$ of patients tested. Interestingly, results from our own group (HIM Davidson, $\mathrm{R}$ Pattison and RA Richardson, unpublished results) also indicate an apparent lowering of bitter TDT in end-stage cancer patients when compared with age-matched controls, and that this aberration is more common in patients with weight loss.

However, the literature in this area is not entirely consistent. Ovesen et al. (1991) reported higher TDT in patients with cancer than in a healthy control group. This effect appeared to reverse following successful treatment from a variety of chemotherapeutic regimens. They concluded that this indicated a role for the disease process in increasing taste thresholds. However, it may equally suggest a sensitization of afferent nerves by the therapeutic agents used, some of which are known to be neuroactive (e.g. vinca alkaloids). In addition, TDT were not determined chemically, but by electrical gustometry. This methodology could be criticized in that it may mask subtle changes in initiation of activity in gustatory neural pathways and transduction processes which 
could be present in specific taste modalities in neoplastic disease.

\section{Could the taste pathway be sensitized?}

The presence of afferent neural connections that project to the brain stem (in the region of the nucleus of the solitary tract) from the tongue and other regions of the gut has been known for some time (Hermann et al. 1983). The convergence of this gustatory and gastrointestinal vagal afferent information in the brain occurs at the level of the parabrachial nucleus and plays an important role in the determination of taste preference (Hermann \& Rogers, 1985). The presence of circulating compounds such as cytokines or other inflammatory mediators may have the ability to activate gustatory afferents, as has been demonstrated in hepatic vagal afferents (Niijima \& Meguid, 1995). This could produce sensitization of afferent information, resulting in both enhanced sensation of particular receptor or ion channel-mediated taste modalities, and hence inappropriate perception which manifests as alterations in taste preferences or thresholds.

There are several other mechanistic pathophysiological parallels in which sensitization of visceral information from the periphery results in apparently inappropriate central perception. For example, the occurrence of pregnancy sickness has been suggested to be due to altered hormonal levels which sensitize the area postrema. This is the area of the brain which receives inputs from the visceral afferents and can stimulate and modulate the emetic pathway (Andrews \& Whitehead, 1990). Likewise, the hyperalgesia induced by prostaglandins and other inflammatory mediators during trauma causes sensitization of the nociceptive afferents and effectively reduces their threshold for activation.

A case of 'phantom taste' reported by Miller \& Bartoshuk (1991) reinforces the hypothesis that abnormal neuronal activity may cause aberrant taste perception. When the right chorda tympani nerve of a patient was removed she subsequently experienced a salty taste in her mouth. This perception was not due to stimulation of taste buds, as taste testing with salt solution placed on the tongue revealed no perception of the solution and the normal neural connections to the brain had been removed. The authors concluded that it was abnormal stimulation in the severed nerve causing salt taste to be perceived without the normal stimulus present; i.e. the afferent nerve was stimulated without depolarization of sensory receptors (taste receptor cells) or normal transduction mechanisms. There are other pathophysiological parallels for this, particularly in neuropathic pain such as that which occurs in amputees. The patient feels pain in the amputated region because the afferent end of the nerves are still present and abnormal spontaneous evoked potentials occur mimicking the painful nociceptive stimulus from the area which has been amputated.

Recent electrophysiological evidence from recordings of the chorda tympani in animals following lipopolysaccharide injection also suggest that the products of immune cells can modulate the peripheral gustatory system (Phillips \& Hill, 1996). The supersensitive responses seen in this study may be paralleled with the low thresholds for certain taste modalities found by a number of studies in patients with cancer (DeWys \& Walters, 1975; Williams \& Cohen, 1978; Pattison et al. 1997). The circulating cytokine levels and acute-phase proteins which are associated with weight-losing cancer patients are evidence of activation of the immune system and the presence of an on-going inflammatory response in the disease (Falconer et al. 1994). It is not outside the bounds of possibility that these may have some bearing on the dysgeusia apparent in some cancer patients. Most studies in cancer patients implicate tumour necrosis factor- $\alpha$ and interleukin- $1 \beta$ and -6 in orchestrating the mechanisms of anorexia and cachexia (Souba, 1994). This response in human subjects to the presence of neoplastic disease is analogous to the profile of immunomodulators released in animals when subjected to lipopolysaccharide (endotoxin) injection.

In studies from our own group (Pattison et al. 1997) results from measurements in end-stage cancer patients revealed altered taste thresholds with respect to the bitter modality, and were most apparent in those who were losing weight. The bitter thresholds were lower than in an agematched group, suggesting an increased sensitivity to bitter. Patients with low thresholds to bitter had an on-going metabolic response, as indicated by higher levels of the acute-phase protein $\mathrm{C}$-reactive protein. In addition, biochemical analysis of their plasma cytokine profiles (interleukin-1 $\beta$, tumour necrosis factor- $\alpha$ and interleukin-6) showed that patients with the lowest bitter thresholds had the highest levels of tumour necrosis factor- $\alpha$. This suggests that there is an association between weight loss and mediators of this catabolic state in cancer patients, and implicates immune cell-derived products in peripheral modulation of the gustatory system; at least for one specific taste modality.

In the same study (Pattison et al. 1997), odour discrimination was tested in these end-stage patients. When confronted with a number of household odours the cancer group recognized significantly more than the age-matched group (Fig. 1), suggesting a heightened olfactory perception in addition to lower bitter thresholds.

Weight loss in undiagnosed patients with cancer is usually one of the main initial factors for seeking medical advice. In our own study there appeared to be an association with the appearance of weight loss and taste changes. Of the cancer patients recruited, $39 \%$ reported the onset of taste changes at

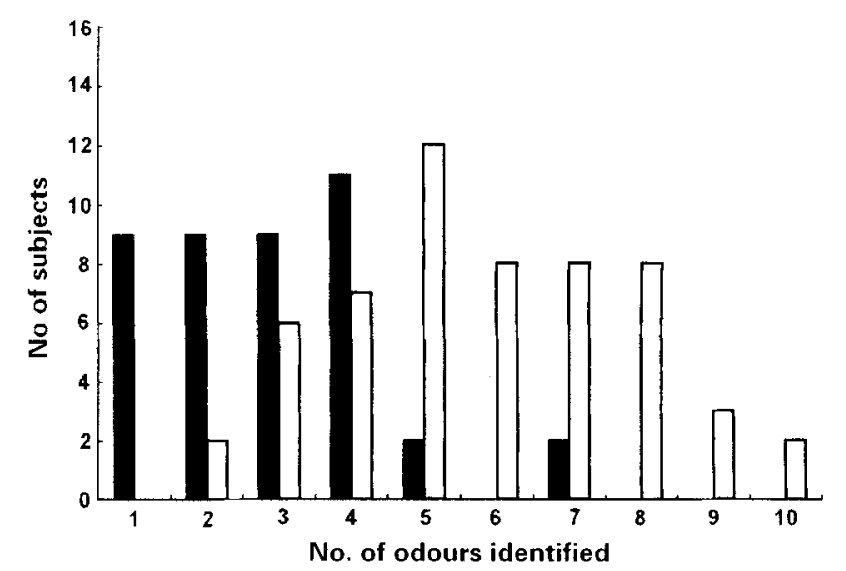

Fig. 1. Odour discrimination in end-stage cancer patients ( $\square$ ) and age-matched controls ( $(\boldsymbol{)})$. 
about the time of diagnosis. However, taste changes and weight loss are by no means the only symptoms which are associated with the presence of neoplastic disease. Many gastrointestinal-mediated symptoms are evident, including early satiety, nausea and vomiting, and abdominal fullness. The presence of these associated symptoms is not dependent on chemotherapy or radiotherapy regimens, but they do appear most commonly in patients with weight loss (Grosvenor et al. 1989). These visceral sensations are all mediated through the so-called gut-brain axis, and are perceived after stimulation of, or the presence of abnormal spike frequency in, the mainly vagal afferent nerves. In conditions such as cancer where there is an on-going acute-phase response resulting in significant weight loss (Fearon, 1992), there may be a general sensitization of visceral afferent information. The implications of this with respect to food intake would most probably be a reduction in energy intake because of reduced thresholds for normal post-ingestive- and postabsorptive-derived gut stimuli (see Fig. 2). This would be compounded by alterations in taste, particularly the bitter modality, with the increased likelihood of inducing food aversions.

If the sensitization of gustatory (and other afferents) is mediated through cytokine action it may be possible to attenuate their action and prevent these mediators orchestrating cachexia, and also reduce the impact of an on-going inflammatory response on chemosensory function in the disease and thus improve energy intake which even in advanced stages of the disease may improve quality of life.

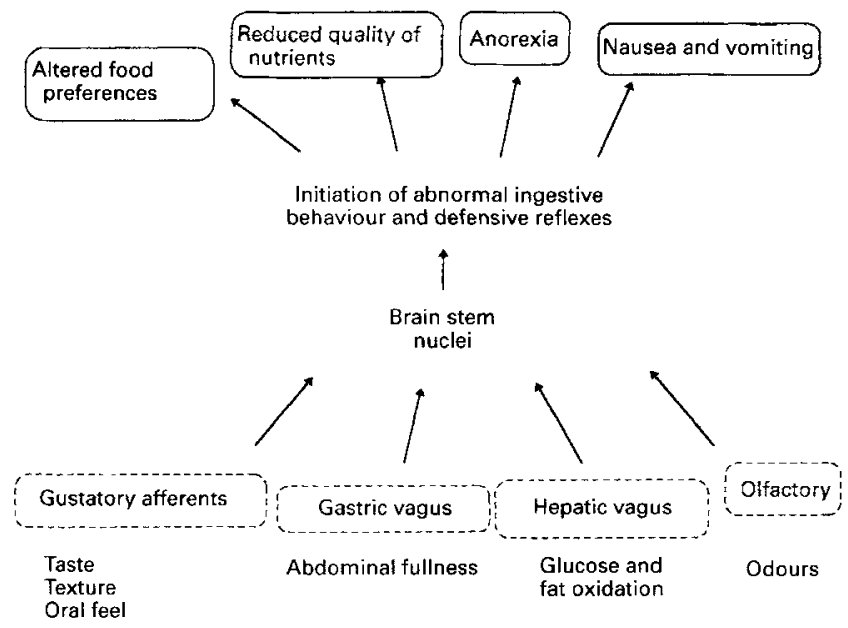

Fig. 2. Possible effects of gut-derived afferent sensitization on food selection and intake.
This is by no means a novel concept. Attenuation of the production of cytokines (interleukin-1 $\beta$ and tumour necrosis factor- $\alpha$ ) has been shown to occur in vitro after fish oil supplementation (Endres et al. 1989). This novel method of nutritional pharmacology has been used in a number of clinical disorders (for review, see Sanders, 1993) with varying degrees of success. In patients with significant and rapid weight loss (5 kg/month), as can occur in pancreatic cancer, remarkable reversal of this occurs with fish oil supplementation (Wigmore et al. 1996). Recently, our own group have assessed the effects of fish oil supplementation on TDT in end-stage cancer patients subjectively reporting taste changes. Results at present (HIM Davidson, R Pattison and RA Richardson, unpublished results) are equivocal, but this type of nutritional support may provide relief of aberrant gustatory changes in a population where they disproportionately impinge on quality of life.

\section{Taste aberrations in liver disease}

The prevalence of gustatory aberration in liver disease is well recognized (Table 2 ). In addition to dysgeusia, dysosmia has also been reported in patients with liver disease (Henkin \& Smith, 1971). Of course, with the functional capacity of this metabolic organ at the centre of nutrient metabolism compromised to such an extent, it is not surprising that with hepatic disease chemosensory systems that influence nutrient intake are affected; nor is the fact that undernutrition is prevalent in this population an unexpected finding (Morgan \& Levine, 1986; Marsano \& McClain, 1991). The question of whether it is the pathology which alters normal gustatory function and hence compromises nutritional status by affecting intake, or poor nutritional state which adversely affects taste perception, remains open to debate.

There does not appear to be the cytokine orchestration of weight loss in liver disease that appears in cancer cachexia. The chemosensory aberrations which occur in liver disease are likely to be due to mechanisms other than gustatory immunomodulation. However, there is increasing evidence to suggest that the liver plays a central role in ingestive behaviour (Scharrer \& Langhans, 1988) and has the ability to influence feeding behaviour, including food preference, through hepatic afferent discharge modulation by absorbed nutrients or metabolites of hepatic fuel oxidation (Tordoff et al. 1990; Niijima \& Meguid, 1995).

Certainly it is evident that bizarre food preferences occur in the presence of chronic liver disease, and that some patients find certain foods palatable that most may even find abhorrent. Deems et al. (1991) reported patients with

Table 2. Changes in taste and food preferences occurring in liver damage

\begin{tabular}{|c|c|c|c|c|}
\hline Patient group & Chemosensory disorder & Food preference reported & Associated nutrient deficiency & Reference \\
\hline Cirrhotics & $\begin{array}{l}\text { Reduced taste and } \\
\text { olfactory thresholds }\end{array}$ & Not measured & Not related to $\mathrm{Zn}, \mathrm{Cu}, \mathrm{Mg}, \mathrm{Ca}, \mathrm{Se}$ & Burch et al. (1978) \\
\hline Cholestatic & & $\begin{array}{l}\text { Low sweet and salt } \\
\text { Low sour and bitter }\end{array}$ & Not measured & Deems \& Friedman (1988) \\
\hline & & Low fat & Not measured & Deems \& Friedman (1988) \\
\hline Cholestatic disease & $\begin{array}{l}\text { Low taste acuity by } \\
\text { electrogustometry }\end{array}$ & Not measured & RBP & Imamine et al. (1990) \\
\hline Alcoholic cirrhotics & $\begin{array}{l}\text { High TDT } \\
\text { Low olfactory score }\end{array}$ & None detected & $\begin{array}{l}\text { Not vitamin } A, Z n, \alpha \text { - or } \beta \text {-carotene } \\
\text { Mg associated with salt }\end{array}$ & Madden et al. (1997) \\
\hline
\end{tabular}

RBP, retinol-binding protein; TDT, taste detection threshold. 
liver disease preferring and even craving for grapefruit. Our own research (RA Richardson and D McGregor, unpublished results) includes the rather bizarre finding that some cirrhotic patients prefer vinegar as a liquid refreshment rather than a condiment. This particular preference has recently been confirmed by Madden et al. (1997), who found a significant increase in the use of vinegar in a group of cirrhotic patients when compared with a healthy control group.

A number of studies have attempted to investigate the cause of these gustatory aberrations and determine the effective component(s). Imamine et al. (1990) reported the association between taste acuity determined by electrogustometry and serum levels of proteins involved in plasma retinol transport. Patients with low retinol-binding protein, holo-retinol-binding protein and transthyretin showed low taste acuity. However, nutritional status was not determined in this study, which may be at least in part responsible for low levels of transport proteins because of the likelihood of malabsorption in a study including patients with obstructive jaundice, some with a diagnosis of pancreatic cancer. Equally however, low levels of vitamin $\mathrm{A}$ in plasma are likely to affect the physiological function of cells, such as taste receptor cells, with a high turnover rate.

Other micronutrients associated with taste have been investigated in this population in an attempt to elucidate the taste impairment. $\mathrm{Zn}$ is one such candidate, and Sturniolo et al. (1992) measured plasma $\mathrm{Zn}$ status and taste thresholds in patients with cirrhotic livers. Whilst plasma $\mathrm{Zn}$ in patients with cirrhosis was reported to be below that of controls, this did not correlate with TDT measured in the patient population. In addition, since supplementation of $\mathrm{Zn}$ failed to significantly reduce TDT, the authors concluded that it was the disease process per se inducing taste abnormalities in this population.

Smith et al. (1976) reported no correlation between gustatory acuity and plasma variables, including vitamin $\mathrm{A}, \mathrm{Zn}$, retinol-binding protein, bilirubin or prealbumin. The patients under study had chronic or acute liver disease (mainly hepatitis), and results appeared uniform for both groups, suggesting that alterations in taste were due to disordered hepatic function. This is reinforced by the fact that in those patients where their disease resolved the changes in taste did likewise. A recent paper by Madden et al. (1997) measured taste acuity in cirrhotic patients and attempted to correlate these with a number of circulating plasma variables, including $\mathrm{Mg}, \mathrm{Zn}$, vitamin $\mathrm{A}$ and $\alpha$ - and $\beta$-carotene. Although all plasma indicators were significantly lower than those in a healthy control group, only $\mathrm{Mg}$ correlated with salt detection thresholds.

It is apparent that there is no single biochemical variable which contributes to the gustatory aberrations which occur in liver disease, and which as a consequence may provide the basis for food preferences which are evident in this patient population. As such, the solution to re-establishing normal taste remains, and may prevent wholly successful outcomes of nutritional support strategies aimed at this group. Further elucidation of the role of the liver in ingestive behaviour may go some way to identifying effective dietary treatments which are both palatable to these patients and can improve nutritional status.
The pathophysiological processes which occur in chronic liver disease (particularly alcoholic liver disease and primary biliary cirrhosis) are usually so extensive as not to allow the organ to recover from the insult. In many cases the prognosis is such that the only effective therapy is an orthotopic liver transplant. Here the nutritional problems of some recipients of an orthotopic liver transplant are reciprocal to those in end-stage disease. Weight gain is common (Palmer et al. 1991), and the increase in body mass is thought to be almost exclusively due to fat (Davidson et al. 1998). Our own research provides anecdotal evidence suggesting these patients have distressing and occasionally insatiable appetites, contributing to hyperphagia and weight gain. This is supported by Belle et al. (1997) who suggest that these changes in appetite after an orthotopic liver transplant may be particularly distressing and impinge on quality of life. It is easy to speculate that these changes in motivation to eat may be dictated by the loss of hepatic-derived satiety signals which fail to terminate the meal for any substantial amount of time. However, this is again an area where a more fundamental understanding of the hepatic influence on ingestive behaviour may prove pivotal in nutritional management.

\section{References}

Andrews PLR \& Whitehead S (1990) Pregnancy sickness. News in Physiological Sciences 5, 5-10.

Belle SH, Porayko MK, Hoofnagle JH, Lake JR \& Zetterman RK (1997) Changes in quality of life after liver transplantation among adults. Liver Transplantation Surgery 3, 93-104.

Bernard RA \& Halpern BP (1968) Taste changes in vitamin A deficiency. Journal of General Physiology 52, 444-464.

Burch RE, Sackin DA, Urside JA, Jetton MM \& Sullivan JF (1978) Decreased taste and smell acuity in cirrhosis. Annals of Internai Medicine 138, 743-746.

Cohen IK, Schechter PJ \& Henkin RI (1973) Hypogeusia, anorexia and altered zinc metabolism following thermal burn. Journal of the American Medical Association 223, 914-916.

Davidson HIM, Richardson RA, Maytum A, Hinds A, McGilchrist A \& Garden OJ (1998) Body composition changes following orthotopic liver transplantation. Proceedings of the Nutrition Society 58 (In the Press).

Deems RO \& Friedman MI (1988) Macronutrient selection in an animal model of cholestatic liver disease. Appetite 11, 73-80.

Deems RO, Friedman MI, Friedman LS \& Maddrey WC (1991) Clinical manifestations of olfactory and gustatory disorders associated with hepatic and renal disease. In Smell and Taste in Health and Disease, pp. 805-816 [TV Gatchell, editor]. New York, NY: Raven Press.

DeWys WD \& Walters K (1975) Abnormalities of taste sensation in cancer patients. Cancer 36, 1888-1896.

Endres S, Ghorbani R \& Kelley V (1989) The effect of dietary supplementation with $n-3$ polyunsaturated fatty acids on the synthesis and production of IL 1 and TNF alpha by mononuclear cells. New England Journal of Medicine 320, 266-272.

Falconer JS, Fearon KCH, Plester CE, Ross JA \& Carter DC (1994) Cytokines, the acute phase response and resting energy expenditure in cachectic patients with pancreatic cancer. Annals of Surgery 219, 325-331.

Fearon KCH (1992) The mechanisms and treatment of weight loss in cancer. Proceedings of the Nutrition Society 51, 251-261.

Giacosa A, Frascio F, Sukkar SG \& Roncella S (1996) Food intake and body composition in cancer cachexia. Nutrition 12, Suppl. 1, S20-S23. 
Grosvenor M, Bulcavage L \& Chlebowski R (1989) Symptoms potentially influencing weight loss in a cancer population. Cancer 63, 330-334.

Hambidge KM, Hambidge C \& Jacobs MA (1972) Low levels of zinc in hair, anorexia, poor growth and hypogeusia in children. Pediatric Research 6, 868-874.

Henkin RI \& Smith FR (1971) Hyposmia in acute viral hepatitis. Lancet i, 823-826.

Hermann GE, Kohlerman HJ \& Rogers RC (1983) Hepatic-vagal and gustatory afferent interactions in the brainstem of the rat. Journal of the Autonomic Nervous System 9, 477-495.

Hermann GE \& Rogers RC (1985) Convergence of vagal and gustatory afferent input within the parabrachial nucleus of the rat. Journal of the Autonomic Nervous System 13, 1-17.

Imamine $T$, Okuno $M$, Morikawa $H$, Ninomiya $M$, Nishiwaki $S$, Noma A \& Muto $Y$ (1990) Plasma retinol transport system and taste acuity in patients with obstructive jaundice. Gastroenterologica Japonica 25, 206-211.

Madden A, Bradbury W \& Morgan M (1997) Taste perception in cirrhosis: its relationship to circulating micronutrients and food preferences. Hepatology 26, 40-48.

Marsano L \& McClain CJ (1991) Nutrition and alcoholic liver disease. Journal of Parenteral and Enteral Nutrition 15, 337-344.

Miller IJ \& Bartoshuk LM (1991) Taste perception, taste bud distribution and spatial relationships. In Smell and Taste in Health and Disease, pp. 205-233 [TV Getchell, editor]. New York, NY: Raven Press.

Morgan MY \& Levine JA (1986) Nutritional management of patients with liver disease. Journal of Clinical Nutrition and Gastroenterology 1, 303-314.

Mott AE \& Leopold DA (1991) Disorders in taste and smell. Medical Clinics of North America 75, 1321-1353.

Niijima A \& Meguid MM (1995) An electrophysiological study on amino acid sensors in the hepato-portal system in the rat. Obesity Research 3, 714S-745S.

Ohara I, Tabuchi R, Kimura M \& Itokawa Y (1994) Preference for sodium chloride is reduced in protein-deprived juvenile rats. Journal of Nutrition 124, 901-905.

Ohara I, Tabuchi R, Kimura M \& Itokawa Y (1995) Decline of taste sensitivity in protein deficient adult rats. Physiology and Behaviour 57, 921-926.

Osaki T, Ohshima M, Tomita Y, Matsugi N \& Nomura Y (1996) Clinical and physiological investigations in patients with taste abnormality. Joumal of Oral Pathology and Medicine 25, 38-43.
Ovesen L, Sorensen M, Hannibal J \& Allingstrup L (1991) Electrical taste detection thresholds and chemical smell detection thresholds in patients with cancer. Cancer 68, 2260-2265.

Palmer M, Schaffner F \& Thung S (1991) Excessive weight gain after liver transplantation. Transplantation 51, 797-800.

Pattison RM, Dougan H, Richardson RA \& Davidson HIM (1997) Biochemical correlates of altered taste perception in patients with advanced cancer. Clinical Nutrition 16, Suppl. 2, 29.

Phillips LM \& Hill DL (1996) Novel regulation of peripheral gustatory function by the immune system. American Journal of Physiology 271, R857-R862.

Sanders TAB (1993) Marine oils: metabolic effects and role in human nutrition. Proceedings of the Nutrition Society 52, $457-472$.

Scharrer E \& Langhans W (1988) Metabolic and hormonal factors controlling food intake. International Journal of Vitamin and Nutrition Research 58, 249-261.

Schiffman S (1994) Changes in taste and smell: drug interactions and food preferences. Nutrition Reviews 52, S11-S14.

Smith FR, Henkin RI \& Dell RB (1976) Disordered gustatory acuity in liver disease. Gastroenterology 70, 568-571.

Souba W (1994) Cytokine control of nutrition and metabolism in critical illness. Current Problems in Surgery 7, 579-643.

Sturniolo GC, D'Inca R, Parisi G, Giacomazzi F, Montino MC, D'Odorico A, Soranzo P \& Naccarato R (1992) Taste alterations in liver cirrhosis: Are they related to zinc deficiency? Journal of Trace Elements and Electrolytes in Health and Disease 6 , 15-19.

Tordoff MG, Ulrich PM \& Sandler F (1990) Flavor preferences and fructose: Evidence that the liver detects the unconditioned stimulus for the calorie-based learning. Appetite 14, 29-44.

Vazquez M, Pearson PB \& Beachamp GK (1982) Flavour preferences in malnourished Mexican infants. Physiology and Behaviour 28, 513-519.

Vickers ZM, Nielsen SS \& Theologides A (1981) Food preferences of patients with cancer. Journal of the American Dietetic Association 79, 441-445.

Wigmore SJ, Ross JA, Falconer SJ, Plester CE, Tisdale MJ, Carter DC \& Fearon KCH (1996) The effect of polyunsaturated fatty acids on the progression of cachexia in patients with advanced cancer. Nutrition 12, Suppl., S27-S30.

Williams LR \& Cohen MH (1978) Altered taste thresholds in lung cancer. American Journal of Clinical Nutrition 31, $122-125$. 